\title{
Analysis of the relationship between prescribed dose and dosimetric advantage of real-time intraoperatively built custom-linked seeds in iodine-125 prostate brachytherapy
}

Katsumi Hirose ${ }^{1,3}$, Masahiko Aoki ${ }^{1 *}$, Mariko Sato ${ }^{1}$, Hiroyoshi Akimoto ${ }^{1}$, Yasuhiro Hashimoto ${ }^{2}$, Atsushi Imai ${ }^{2}$, Noritaka Kamimura ${ }^{2}$, Hideo Kawaguchi', Yoshiomi Hatayama' ${ }^{1}$ Ichitaro Fujioka', Mitsuki Tanaka', Chikara Ohyama $^{2}$ and Yoshihiro Takai ${ }^{1,3}$

\begin{abstract}
Background: The purpose of this study was to investigate the differences in the dosimetric advantage of using intraoperatively built custom-linked (IBCL) seeds between permanent iodine-125 (I-125) seed implantation (PI) alone and PI followed by external-beam radiation therapy (EBRT) for prostate cancer.

Methods: We reviewed the records of 62 patients with localized prostate cancer who received transperineal interstitial brachytherapy with I-125 using free seeds or IBCL seeds. Twenty-four low- and intermediate-risk patients underwent PI alone with the prescribed dose of $160 \mathrm{~Gy}$, and 39 high-risk patients underwent PI with $110 \mathrm{~Gy}$, followed by EBRT with 45 Gy (PI + EBRT). Intraoperative and post-implant dosimetric parameters 1 month after implantation were collected and analyzed.
\end{abstract}

Results: The numbers of patients implanted with free seeds and IBCL seeds were 14 (58.3\%) and 10 (41.7\%), respectively, in the PI group and 25 (65.8\%) and 13 (34.2\%), respectively, in the PI + EBRT group. In the PI group, although there were significant differences in prostate V100 $(p=0.003)$ and D90 $(p=0.009)$ and rectum V100 $(p=0.026)$ on intraoperative dosimetry, these differences were not found on post-implant dosimetry. In the PI + EBRT group, the dosimetric parameters of IBCL seeds, such as prostate V200 ( $p=0.013)$ and V250 $(p=0.010)$ and urethra D30 $(p=0.038)$, were better than those of free seeds on intraoperative dosimetry. Furthermore, even on post-implant dosimetry, prostate D90 $(p=0.004), \mathrm{V} 150(p=0.001)$, and homogeneity index $(H I, p=0.001)$, as well as V200 $(p=0.001)$ and V250 $(p=0.020)$, and urethra D5 $(p=0.008)$ as well as D30 $(p=0.003)$ had a better dosimetric quality in IBCL seeds than in free seeds. There was no significant difference in the operation time between free seeds and IBCL seeds in each PI and PI + EBRT group.

Conclusions: Our results reveal that greater dosimetric benefits could be obtained using IBCL seeds in the case of permanent implantation with a lower prescribed dose, such as PI + EBRT, rather than PI alone.

Keywords: Prostate cancer, Brachytherapy, IBCL seed, Seed migration

\footnotetext{
*Correspondence: maoki@hirosaki-u.ac.jp

'Department of Radiology and Radiation Oncology, Hirosaki University

Graduate School of Medicine, 5 Zaifu-cho, Hirosaki, Aomori 036-8562, Japan

Full list of author information is available at the end of the article
} 


\section{Background}

Permanent iodine-125 (I-125) seed implantation is a wellestablished curative treatment option for localized prostate cancer and can deliver a high, localized radiation dose to the tumor with excellent biochemical control of disease $[1,2]$. Prostate brachytherapy also has advantages over external beam radiation therapy (EBRT) because of its ability to overcome problems of organ movement. The techniques of seed implantation have been developed in various ways, for example, planning methods (intraoperative real-time planning or inverse planning) and the type of seeds (free or stranded). The difficulty in maintaining the dosimetric quality differs along with the condition of the patient. To confirm the actual dose delivered or identify any deviation from the treatment plan, postoperative dosimetry is recommended [3]. Compared with free seeds, stranded seeds significantly decrease the incidence of seed migration [4-6]. Each type of seed also has identical characteristics in terms of dose quality and distribution. Zaulus et al. first reported that the delivery system with intraoperatively built custom-linked (IBCL) seeds allows the stable implantation of seeds with less seed migration [7]. Ishiyama et al. revealed that dosimetric change due to the selection of seed type with free and IBCL seeds made an impact on the clinical outcome of the study [8].

Recently, combined therapy with lower-prescribed-dose prostate permanent I-125 seed implantation (PI) and EBRT, involving 110-Gy PI followed by 45-Gy EBRT (PI + EBRT), resulting in a total biological effective dose with an $\alpha / \beta$ value of $2 \mathrm{~Gy}\left(\mathrm{BED}_{2}\right)=220-240 \mathrm{~Gy}$, is often performed in patients with high-risk prostate cancer and has shown better biochemical control [1, 9-11]. Along with constant standardization of radioactivity per seed, PI with a higher prescribed dose requires a higher total seed number to achieve an adequate dose quality for the prostate. On the other hand, in PI for high-risk prostate cancer, a comparatively higher peripheral seed number is required to be arranged near the capsule of the prostate because of the risk of tumor relapse from the prostate periphery. As a result, 110-Gy PI with EBRT in high-risk patients might require a greater deal of technical implantation skills than 160-Gy PI in intermediate- and low-risk patients.

Therefore, there is the possibility that the impact of seed type, such as free seeds or stranded seeds, on dose distribution varies according to a dose prescription or a seed number required in each case. However, to the best of our knowledge, the dosimetric advantage conferred by intraoperative-built custom-linked seeds has not been fully elucidated for brachytherapy with a lower prescribed dose similar to that in PI + EBRT cases. In the current analysis, we evaluated the differences in the impact of IBCL seeds on dose quality between two different prescribed doses: 110 Gy in PI + EBRT and 160 Gy in PI on transperineal interstitial prostate brachytherapy.

\section{Methods}

A total of 63 patients with newly diagnosed, localized prostate cancer of clinical stage T1c-T3a with prostatespecific antigen (PSA) levels of $4.0-89.1 \mathrm{ng} / \mathrm{mL}$ and a Gleason score (GS) of 6-9 were treated using transperineal interstitial prostate brachytherapy with radioactive I125 from November 2012 to October 2014. Of these patients, 39 with high-risk prostate cancer were treated with 110-Gy PI, followed by EBRT 1 month after implantation $(\mathrm{PI}+\mathrm{EBRT})$, and 24 with low- and intermediate-risk were treated with 160-Gy PI alone. Implantations for the PI + EBRT group with free seeds were conducted as a cohort study, and a portion of the records were extracted with regards to the period during which medical staffs of a specific composition joined PI for this study. For comparison with our conventional technique with free seeds and the first experience of the use of IBCL seeds, these patients' records were retrospectively reviewed during the specific period in which the learning curve of prostate brachytherapy in our institution had already reached an equilibrium state. Each implantation was conducted by the same composition of medical staff. This study was approved by our institutional review board. All patients were included in this analysis except for one patient in the PI + EBRT group who was excluded because of a partial deficit in clinical data.

All patients also underwent neoadjuvant hormonal therapy. The patient and treatment characteristics are shown in Table 1. One month before implantation, preoperative planning was performed with sagittal transrectal ultrasound images taken in the range of $120^{\circ}-140^{\circ}$ at an angle of $1^{\circ}$ each, which were captured by a treatment planning system $\left(\right.$ SPOTPRO $^{\mathrm{TM}}$, Nucletron, Veenendaal, Netherlands). The entire prostate, urethra along with a balloon catheter, and rectal anterior wall were contoured using reconstructed axial images at $2.5-\mathrm{mm}$ intervals. The clinical target volume (CTV) was defined as the prostate with no margin beyond the organ. The number of seeds for treatment with each prescribed dose was then determined. Patients in the low- and intermediate-risk group received a total dose of 160 Gy. Patients in the high-risk group received a total dose of $110 \mathrm{~Gy}$, followed by 45 Gy EBRT 1 month after implantation. Treatment planning with 160 Gy or 110 Gy was performed using a real-time peripheral loading approach without inverse optimization. The other dosimetric targets were used for prostate implants as follows: the minimal dose received by $90 \%$ of the CTV (D90) $>100 \%$ of the prescribed dose, irradiated dose to $30 \%$ of the urethral volume (UD30) $\leq$ 200 Gy for $160-\mathrm{Gy}$ and $10 \%$ of the urethral volume (UD10) $\leq 200$ Gy for 110-Gy brachytherapy, and rectal volume receiving the prescribed dose $(\mathrm{RV} 100)<1 \mathrm{~cm}^{3}$ for both prescribed doses. In the period from November 2012 to February 2014, the patients received OncoSeed ${ }^{\mathrm{TM}} \mathrm{I}-125$ 
Table 1 The patient and treatment characteristics $(n=62)$

\begin{tabular}{|c|c|c|c|c|c|c|}
\hline \multirow[t]{2}{*}{ Characteristics } & \multicolumn{3}{|l|}{ PI group } & \multicolumn{3}{|c|}{$\mathrm{PI}+\mathrm{EBRT}$ group } \\
\hline & Free seed & $\mathrm{IBCL}$ seed & $p$ value & Free seed & $\mathrm{IBCL}$ seed & $p$ value \\
\hline Patients & 14 & 10 & & 25 & 13 & \\
\hline Age (years) & $70.5 \pm 5.9$ & $72.5 \pm 6.4$ & 0.859 & $73 \pm 4.1$ & $735 \pm 4.5$ & 0.093 \\
\hline Intra-operative prostate volume $\left(\mathrm{cm}^{3}\right)$ & $18.9 \pm 4.7$ & $18.5 \pm 10.6$ & 0.595 & $18.9 \pm 7.7$ & $16.7 \pm 9.7$ & 0.734 \\
\hline Post-operative prostate volume $\left(\mathrm{cm}^{3}\right)$ & $16.4 \pm 5.6$ & $17.8 \pm 9.7$ & 0.507 & $16.4 \pm 6.9$ & $16.5 \pm 10.0$ & 0.326 \\
\hline Initial PSA (ng/mL) & $7.1 \pm 3.1$ & $8.9 \pm 4.6$ & 0.344 & $8.8 \pm 16.4$ & $13.4 \pm 27.9$ & 0.424 \\
\hline Clinical stage & & & $N A$ & & & 0.077 \\
\hline T1c-T2a & 12 & 9 & & 11 & 5 & \\
\hline $\mathrm{T} 2 \mathrm{~b}$ & 2 & 1 & & 3 & 6 & \\
\hline T2c-T3a & 0 & 0 & & 11 & 2 & \\
\hline Gleason sum & & & $N A$ & & & NA \\
\hline$\leq 6$ & 1 & 0 & & 0 & 0 & \\
\hline 7 & 13 & 10 & & 2 & 1 & \\
\hline$\geq 8$ & 0 & 0 & & 23 & 12 & \\
\hline Risk group & & & $N A$ & & & NA \\
\hline Low & 1 & 0 & & 0 & 0 & \\
\hline Intermediate & 13 & 10 & & 0 & 0 & \\
\hline High & 0 & 0 & & 25 & 13 & \\
\hline Implanted seed number & $65.5 \pm 8.8$ & $63.5 \pm 11.2$ & 0.780 & $51 \pm 10.3$ & $47 \pm 9.6$ & 0.318 \\
\hline Total activity (MBq) & $748 \pm 141$ & $715 \pm 250$ & 0.652 & $561 \pm 134$ & $517 \pm 143$ & 0.465 \\
\hline
\end{tabular}

Abbreviations: PSA Prostate-specific antigen

All values were expressed as median \pm standard deviation

implants (GE Healthcare, Medi-Physics Inc., Arlington Heights, IL) as a type of free I-125 seeds using the Mick applicator (Mick Radio-Nuclear Instruments, Mount Vermont, NY), and in the next period from February 2014 to October 2014, the patients received Quicklink ${ }^{\circ}$ I-125 implants as a type of intraoperatively linked I-125 seeds using a Quicklink ${ }^{\circ}$ device (CR Bard, Covington, GA). All types of seeds were manually delivered, and two specific physicians and two physicists were involved in these implantations in an alternating fashion.

Postoperatively, fluoroscopic images of the pelvis were obtained for confirmation of the number of implanted seeds. All patients underwent a series of radiographs, including chest and kidney-ureter-bladder radiographs, to identify the sites of seed loss and migration. For the post-implant dosimetric evaluation, all patients underwent computed tomography scanning of the pelvis with $1.25-\mathrm{mm}$-thick slices the next day and 30 days after implantation. The dosimetric parameters with regard to intraoperative images and images at 30 days after implantation were calculated for dosimetric quality and dose distribution as intraoperative values and postimplant values, respectively, such as prostate D90 (Gy, the radiation dose to $90 \%$ of the prostate volume), V100 (\%, the percentage of the prostate volume receiving $100 \%$ of the prescribed dose), V150 (\%, the percentage of the prostate volume receiving $150 \%$ of the prescribed dose), V200 (\%, the percentage of the prostate volume receiving $200 \%$ of the prescribed dose), V250 (\%, the percentage of the prostate volume receiving $250 \%$ of the prescribed dose), urethra D5 (Gy, the radiation dose to $5 \%$ of the urethral volume), urethra D30 (Gy, the radiation dose to $30 \%$ of the urethral volume), rectum V100 (\%, the percentage of the rectal volume receiving $100 \%$ of the prescribed dose), and $\mathrm{HI}$ (\%, the homogeneity index). V150, V200, and V250 were calculated to evaluate the existence of local "hot spots." HI was calculated for the fraction of the prostate volume receiving between $100 \%$ and $150 \%$ of the prescribed dose as described by Saw et al. [12]. Then, HI was defined as

$$
H I=\frac{V 100-V 150}{V 100} \times 100 \%
$$

Seed migration was also scored when seeds were confirmed to have been localized in the chest and abdomen or separated by $1 \mathrm{~cm}$ from the seed cluster in the pelvis. Seeds placed into the bladder and seminal vesicles were not scored as seed migration but as seed loss.

\section{Statistical analysis}

All parameters were calculated for both groups. Statistical analysis was performed using the $\chi^{2}$ test or Fisher's 
exact test for qualitative variables and the Student's $t$-test or Mann-Whitney U test for quantitative variables, depending on the data distribution. The significance level was set as $p<0.05$.

\section{Results}

As shown in Table 1, the numbers of patients implanted with free seeds and IBCL seeds were 14 (58.3\%) and 10 (41.7\%), respectively, in the PI group and 25 (65.8\%) and 13 (34.2\%), respectively, in the PI + EBRT group. There were no significant differences in patient and treatment characteristics between patients implanted with free seeds and IBCL seeds in each group. However, only for analyses including all seed types in comparisons between the PI and PI + EBRT groups, there were significant differences in the GS $(p<0.001)$, clinical T stage $(p<0.001)$, number of seeds $(64 \pm 9.7$ vs $50 \pm 10.1, p<0.001)$, and total activity $(748 \pm 189 \mathrm{mCi}$ vs $550 \pm 137 \mathrm{mCi}, p<0.001)$ as expected, although the parameter of the D'Amico risk classification was not evaluated because of the extreme data distribution.

In the PI group, no impact of differences in seed type was detected on the parameters of CTV dose distribution. However, only for IBCL seeds, prostate V150 and V200 were decreased on post-implant dosimetry 1 month after implantation compared with intraoperative dosimetry and the changes were significant $(57.3 \pm 11.1 \%$ vs $68.3 \pm 10.3 \%$, with $p=0.006$ for $\mathrm{V} 150 ; 25.7 \pm 6.1 \%$ vs $29.5 \pm 5.7 \%$, with $p=0.030$ for V200, respectively, Fig. 1a). Although the parameter of prostate D90 in cases with IBCL seeds was significantly higher than that in cases with free seeds on intraoperative dosimetry $(209.1 \pm$ 12.6 Gy vs $195.8 \pm 10.3 \mathrm{~Gy}, p=0.009$ ), this difference disappeared on post-implant dosimetry 1 month after implantation (190.1 \pm 15.2 Gy vs $190.3 \pm 23.6$ Gy, $p=$ 0.9783 , Fig. 1b). The values of $\mathrm{HI}$, which indicate the

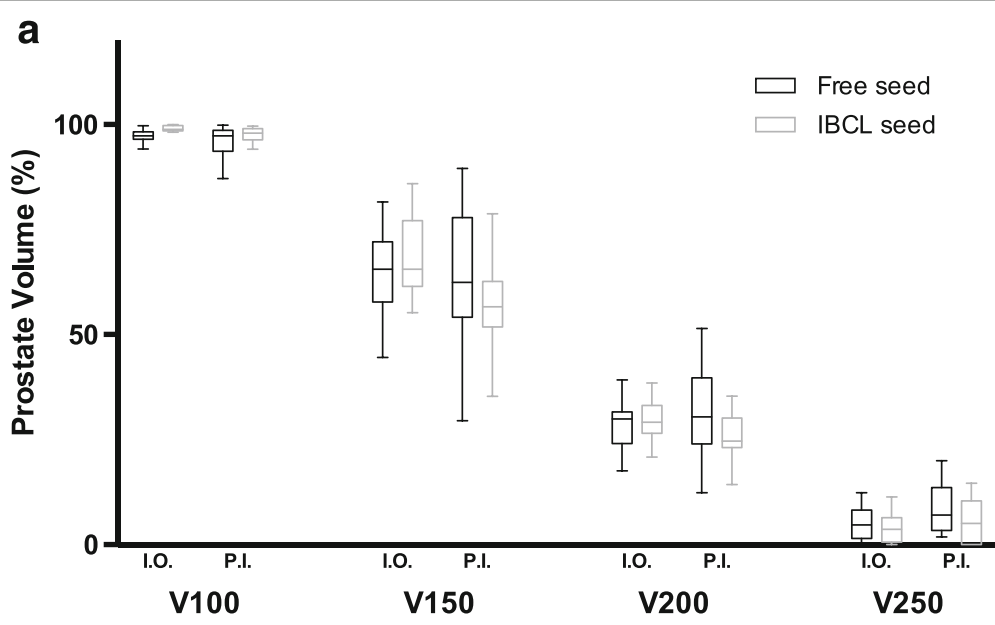

b

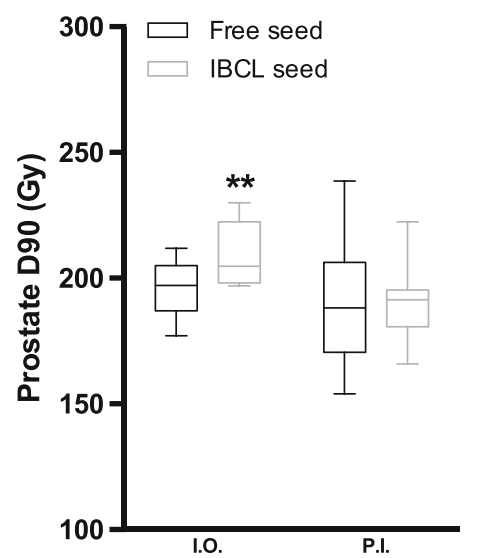

c



Fig. 1 Changes in dosimetric parameters for the PI group with 160-Gy PI. a The percentages of the prostate volume receiving each percentage of the prescribed dose. $\mathbf{b}$ The intraoperative and post-implant D90 prostate values. $\mathbf{c}$ The homogeneity index for free and IBCL seed groups. The spread of the data is denoted by a box and whiskers plot: box limits represent the 25th and 75th percentiles; the line within the box represents the median; whisker ends represent the 1 st and 99 th percentiles. ${ }^{*} p<0.05,{ }^{* *} p<0.01$, in comparison with free seed and IBCL seed in each I.O. and P.I. Abbreviations: I.O. = intraoperative dosimetry; P.I. = postimplant dosimetry 
homogeneity of the CTV dose, did not differ between free and IBCL seeds on either intraoperative dosimetry $(33.9 \pm 10.0$ vs $31.1 \pm 10.0, p=0.509)$ or post-implant dosimetry ( $33.6 \pm 15.5$ vs $41.3 \pm 10.9, p=0.191$, Fig. $1 \mathrm{c})$. For the evaluation of critical organs as shown in Table 2, although a positive effect of the use of free seeds on rectum V100 $(p=0.026)$ was detected, this effect was not seen on post-implant dosimetry $(p=0.119)$. There were no differences in urethra D30 $(p=0.398$, and $p=0.159)$ and D5 ( $p=0.236$, and $p=0.488)$ between these seed types on intraoperative and post-implant evaluation, respectively.

In the PI + EBRT group, the parameters of prostate V200 and V250 in cases with IBCL seeds were significantly improved compared with cases with free seeds intraoperatively $(33.4 \pm 8.6 \%$ vs $40.8 \pm 9.0 \%$ with $p=0.013$ for V200, and $5.7 \pm 5.2 \%$ vs $12.0 \pm 7.2 \% p=0.010$, respectively). These impacts of using IBCL seeds on the improvement of dose distribution became more prominent with fine values of prostate V150 $(62.8 \pm 12.9 \%$ vs $80.2 \pm 10.0 \%, p=0.001), \mathrm{V} 200(28.0 \pm 6.5 \%$ vs $47.5 \pm$ $12.7 \%, p=0.001)$, and V250 ( $8.3 \pm 5.4 \%$ vs $17.4 \pm 12.9 \%$, $p=0.020)$ in post-implant dosimetry on day 30. For IBCL seeds, prostate V150 and V200 were decreased on post-implant dosimetry 1 month after implantation compared with intraoperative dosimetry with significant changes $(62.8 \pm 12.9 \%$ vs $74.0 \pm 12.6 \%$ with $p<0.001$ for V150, and $28.0 \pm 6.5 \%$ vs $33.4 \pm 8.6 \%$ with $p<0.001$ for V200, respectively, Fig. 1a). On the contrary, for free seeds, prostate V200 was increased on post-implant dosimetry compared with intraoperative dosimetry (47.5 $\pm 12.7 \%$ vs $40.8 \pm 9.0 \%, p<0.001$, Fig. $2 a$ ). Although there was no difference in prostate D90 between free seeds and IBCL seeds on intraoperative evaluation (153.0 \pm 11.8 Gy vs $149.1 \pm 9.1 \mathrm{~Gy}, p=0.297$ ), the value was decreased preferentially in the IBCL seed group on postimplant evaluation, resulting in a significant difference $(148.3 \pm 13.7$ Gy vs $134.5 \pm 12.1$ Gy, $p=0.004$, Fig. $2 b)$. The values of HI did not differ between free seeds and IBCL seeds on intraoperative dosimetry $(18.9 \pm 8.8$ vs $25.4 \pm 12.5, p=0.067$ ) but were improved with IBCL seeds on post-implant evaluation $(18.7 \pm 9.3$ vs $35.7 \pm$
11.7, $p<0.001$, Fig. 2c). In the assessment of critical organs as shown in Table 3, although a tendency toward an improvement in rectum V100 was seen in cases with IBCL seeds on intraoperative dosimetry $(p=0.074)$, this effect was diminished on post-implant dosimetry $(p=$ 0.306). The parameters of urethra D30 were significantly improved in cases with IBCL seeds compared with cases with free seeds. These impacts of using IBCL seeds on the improvement of the urethral dose were greater on post-implant dosimetry ( $p=0.003$ for D30, and $p=0.008$ for D5).

One month after implantation, seed migration was detected in $4(28.6 \%)$ and $0(0 \%)$ patients with free seeds and IBCL seeds, respectively, in the PI group and 6 (24.0\%) and $0(0 \%)$ patients, respectively, in the PI + EBRT group.

A record of operation time in one IBCL seed case of the PI group was lost, and the numbers of patients treated with free seeds and IBCL seeds for the analysis of operation time were $14(60.9 \%)$ and 9 (39.1\%), respectively, in the PI group and $25(65.8 \%)$ and 13 (34.2\%), respectively, in the PI + EBRT group. Differences were not detected between cases with free seeds and IBCL seeds in either the PI $(100 \pm 28.3$ vs $105 \pm 17.7$, $p=0.765)$ or the PI + EBRT group $(110 \pm 22.0$ vs $130 \pm$ 13.0, $p=0.097$, Fig. 3).

\section{Discussion}

Intraoperative prostate shape distortion and swelling is generally experienced during permanent brachytherapy of the prostate. These volumetric changes in the prostate are reported by McLaughlin et al. to have an impact on dosimetry of permanent implantation [13]. Although the use of stranded seeds is expected to prevent intraprostatic seed displacement and migration [14, 15], Pinkawa et al. reported that even using stranded seeds generates negligible intraprostatic seed displacement, which correlates with the influence of dosimetry [16]. Chng et al. revealed that intraprostatic seed displacement and unexpected seed misorientation, even using stranded seeds, may also decrease dose quality [17]. Fagundes et al. and Lin et al. reported that stranded seeds improve the

Table 2 The intra-operative and post-implant dosimetric values for critical organs in PI group $(n=24)$

\begin{tabular}{|c|c|c|c|c|c|c|}
\hline \multirow{2}{*}{$\begin{array}{l}\text { Risk } \\
\text { organs }\end{array}$} & \multicolumn{3}{|c|}{ Intra-operative values } & \multicolumn{3}{|c|}{ Post-implant values } \\
\hline & Free seed & $\mathrm{IBCL}$ seed & $\overline{p \text { value }}$ & Free seed & $\mathrm{IBCL}$ seed & $p$ value \\
\hline \multicolumn{7}{|l|}{ Urethra } \\
\hline D30 & $226.9 \pm 23.4$ & $223.7 \pm 14.3$ & 0.398 & $248.9 \pm 35.8$ & $219.9 \pm 23.6$ & 0.159 \\
\hline D5 & $245.7 \pm 37.3$ & $235.1 \pm 15.1$ & 0.236 & $278.6 \pm 44.0$ & $255.6 \pm 46.7$ & 0.488 \\
\hline \multicolumn{7}{|l|}{ Rectum } \\
\hline V100 & $0.07 \pm 0.16$ & $0.30 \pm 0.32$ & 0.026 & $0.14 \pm 0.13$ & $0.23 \pm 0.35$ & 0.119 \\
\hline
\end{tabular}




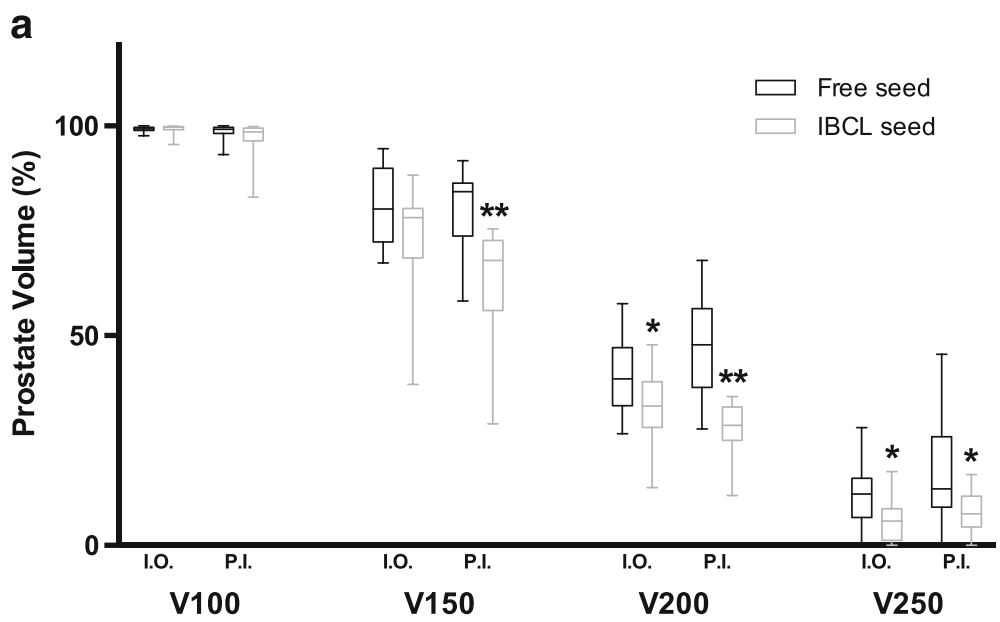

b
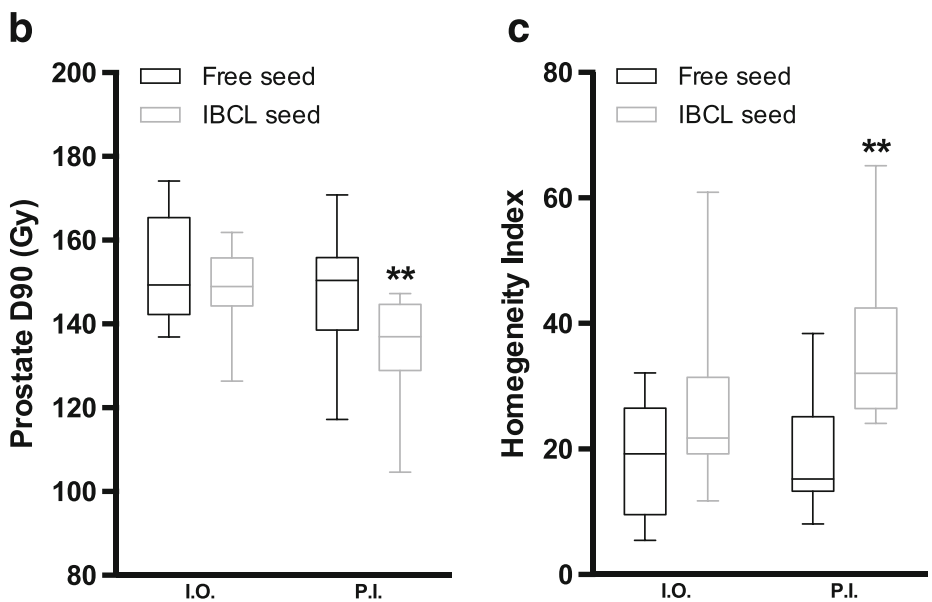

Fig. 2 Changes in dosimetric parameters for the PI + EBRT group with 110-Gy PI. a The percentages of the prostate volume receiving each percentage of the prescribed dose. $\mathbf{b}$ The intraoperative and post-implant D90 prostate values. $\mathbf{c}$ The homogeneity index for free and IBCL seed groups. The spread of the data is denoted by a box and whiskers plot: box limits represent the 25th and 75th percentiles; the line within the box represents the median; whisker ends represent the 1st and 99th percentiles. ${ }^{* *} p<0.01$, in comparison with free seed and IBCL seed in each I.O. and P.I. Abbreviations: I.O. = intraoperative dosimetry; P.I. = postimplant dosimetry

dosimetry of prostate V100 and D90 significantly, compared with free seeds $[18,19]$. In agreement with these results, Heysek et al. presented that D90 was improved by the use of stranded seeds [20]. On the other hand, Salbishkumar reported that dosimetric advantages were not observed at day 7 , although there were advantages of the use of stranded seed in V100 and D90, and a better dose distribution in V150 was also attained with stranded seeds compared with free seeds at day 0 after implantation. [6]. As in these reports, there is no

Table 3 The intra-operative and post-implant dosimetric values for critical organs in PI + EBRT group $(n=38)$

\begin{tabular}{|c|c|c|c|c|c|c|}
\hline \multirow{2}{*}{$\begin{array}{l}\text { Risk } \\
\text { organs }\end{array}$} & \multicolumn{3}{|c|}{ Intra-operative values } & \multicolumn{3}{|c|}{ Post-implant values } \\
\hline & Free seed & $\mathrm{IBCL}$ seed & $p$ value & Free seed & $\mathrm{IBCL}$ seed & $p$ value \\
\hline \multicolumn{7}{|l|}{ Urethra } \\
\hline D30 & $174.5 \pm 14.3$ & $166.0 \pm 14.2$ & 0.038 & $195.1 \pm 32.7$ & $165.2 \pm 23.2$ & 0.003 \\
\hline D5 & $190.9 \pm 19.4$ & $184.4 \pm 20.4$ & 0.29 & $230.1 \pm 36.3$ & $200.3+32.6$ & 0.008 \\
\hline \multicolumn{7}{|l|}{ Rectum } \\
\hline V100 & $0.42 \pm 0.44$ & $0.13 \pm 0.36$ & 0.074 & $0.29 \pm 0.35$ & $0.31 \pm 0.20$ & 0.306 \\
\hline
\end{tabular}

Abbreviations: PI Permanent seed implantation, EBRT External beam radiotherapy, IBCL Intraoperatively built custom-linked All values were expressed as mean \pm standard deviation 


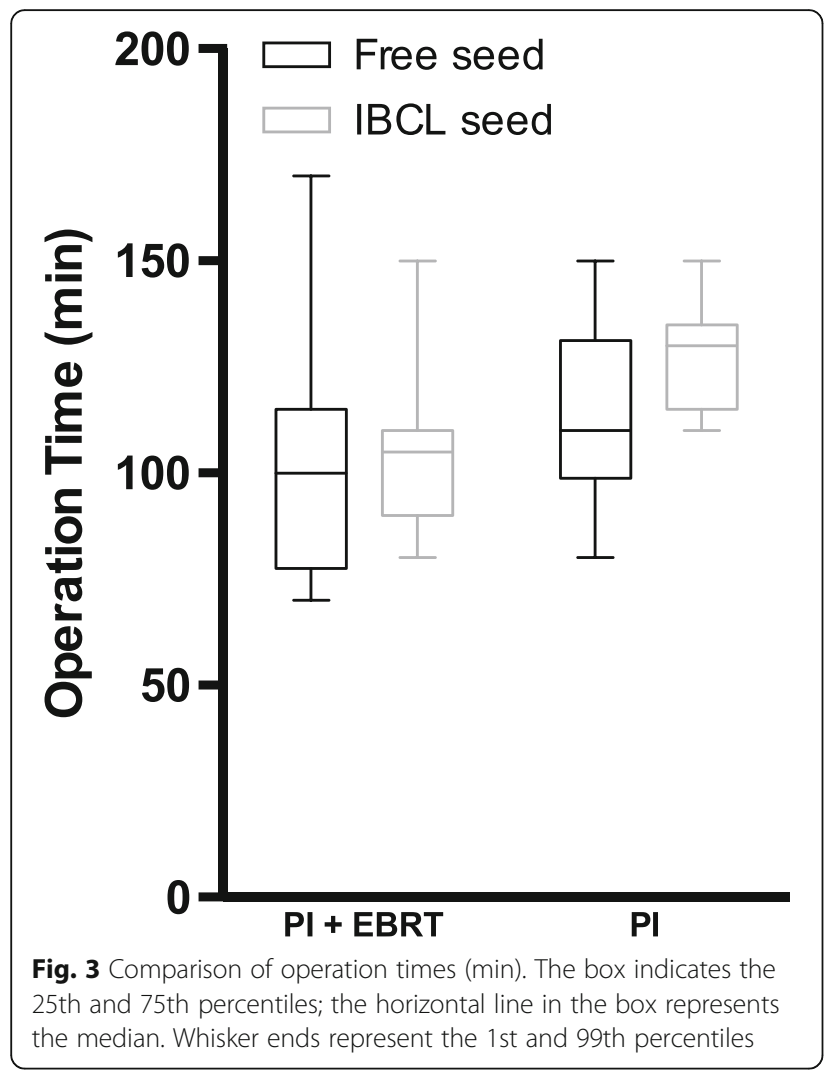

evidence that stranded seeds are necessarily superior to free seeds.

In this study, we are the first to report the dosimetric advantage of intraoperatively real-time custom-linked seeds in PI with a lower prescribed dose. This type of seed was first reported by Zaulus et al. [7]. They confirmed the validity of the use of IBCL seeds with regards to dosimetry in PI prescribed 160 Gy compared with free seeds. Later, Jarusevicius et al. reported in a cohort study with 230 patients that IBCL seeds conferred a dosimetric advantage over free seeds in PI prescribed 160 Gy [21]. However, Ishiyama et al. could not observe the advantage of the use of IBCL seeds in PI prescribed 145 Gy, although IBCL seeds prevented seed migration [8]. In agreement with a previous report, Katayama et al. reported no evidence of improvement in prostate D90, which was observed at day 30 in PI prescribed 144 Gy [22]. Altogether, we can find no evidence in these reports as to whether a dosimetric advantage exists in PI with IBCL seeds. These results suggest that the impact of seed type, such as free or IBCL seeds, on dose quality differs between institutions due to the difference in methodology for placement of the seeds and the shape of the dose distribution. In this study, we adopted a real-time peripheral loading approach without inverse optimization, and achieved the improvement of dosimetry for organs at risk, such as urethra and rectum, as well as the CTV dose homogeneity. I-125 emits low energy photons with average $28 \mathrm{keV}$, which is lower than the photons of EBRT, resulting in limited tissue penetration of photons [23]. Then, effective radiation doses are confined to a few millimeters beyond the target with less dose to peripheral tissues. Therefore, our results imply that brachytherapy, particularly with stranded sources, potentially give larger dose to peripheral margin more safely than EBRT, with less dose to an adjacent organ one wants to avoid (i.e. rectum and bladder) that is not possible with EBRT because of its higher energy.

As mentioned above, even though the use of stranded seeds potentially generates seed displacement [16], the method of real-time treatment plan refinement, such as seed localization and dosimetric modification, is more suitable for uncertainty in PI. Therefore, IBCL seeds, which are real-time built depending on the situation, are theoretically superior to preoperatively built stranded seeds. In this study, we determined that the use of IBCL seeds conferred an advantage on dosimetry in PI with a lower dose followed by EBRT, which is continuously observed until 1 month after implantation but no advantage in PI as a monotherapy with a higher dose. A possible reason for this is that the deficit in dosimetry caused by seed displacement and misorientation might be compensated for easily, even by the use of free seeds, due to the small contribution of each seed to the total radioactivity in PI with a higher dose, and that compensation of the deficit in dosimetry might be accomplished only by the use of IBCL seeds, which might be able to overcome the great challenge caused by the large contribution of each seed to the total radioactivity in PI with a lower dose.

The number of patients reviewed in this study was limited because the specific period was extracted in which prostate brachytherapy in our institution had already reached an equilibrium state in terms of technical skills, and all procedures were conducted by the same composition of medical staff. However, even though few patients were included, the advantage of IBCL seeds was observed; thus, a larger impact of IBCL is expected with regards to PI with a lower dose.

In the present study, no seed migration was observed in the IBCL group. The incidence of seed migration was widely reported as $0-69.4 \%$ in patients treated with $145-$ or 160-Gy PI [4, 5, 24-31]. Especially in lower-dose PI cases, such as those treated with $110 \mathrm{~Gy}$, there are few reports with regards to seed migration [32]. The utilization of stranded seeds is reported to prevent seed migration [4, 5, 29]. As with stranded seeds, it is reported that IBCL seeds also eliminate the occurrence of seed migration. In agreement with these results, from our results showing no migration in the IBCL group, a reduction of the risk of seed embolization is expected. 


\section{Conclusions}

In conclusion, our study shows the dosimetric advantage of IBCL seed implantation compared with free seed implantation, which is more effective in PI prescribed with a lower dose than a higher dose.

\section{Abbreviations}

CTV: Clinical target volume; EBRT: External-beam radiation therapy; GS: Gleason score; HI: Homogeneity index; I-125: lodine-125; IBCL: Intraoperatively built custom-linked; NA: Not applicable; PI: Permanent implantation

\section{Acknowledgements}

Not applicable.

\section{Funding}

This study did not receive any specific funding.

\section{Availability of data and materials}

The datasets supporting the conclusions of this article are included within the article.

\section{Authors' contributions}

Each author participated sufficiently in the work. $\mathrm{KH}$ and MA designed the study and wrote the manuscript. MS contributed to data analysis. $\mathrm{HA}, \mathrm{YH}, \mathrm{Al}$, $\mathrm{NK}$, and CO participated in the treatment and carried out clinical evaluations of patients in follow-up visits. HK and $\mathrm{YH}$ helped in the collection of clinical data. IF and MT helped with writing the manuscript. YT supervised the study. All authors read and approved the final manuscript.

\section{Ethics approval and consent to participate}

All study participants provided informed consent, and we obtained general consent to the research.

\section{Consent for publication}

Not applicable.

\section{Competing interests}

The authors declare that they have no competing interests.

\section{Publisher's Note}

Springer Nature remains neutral with regard to jurisdictional claims in published maps and institutional affiliations.

\section{Author details}

${ }^{1}$ Department of Radiology and Radiation Oncology, Hirosaki University Graduate School of Medicine, 5 Zaifu-cho, Hirosaki, Aomori 036-8562, Japan. 2Department of Urology, Hirosaki University Graduate School of Medicine, 5 Zaifu-cho, Hirosaki, Aomori 036-8562, Japan. ${ }^{3}$ Department of Radiation Oncology, Southern Tohoku BNCT Research Center, 7-10, Yatsuyamada, Koriyama, Fukushima 963-8052, Japan.

Received: 24 February 2017 Accepted: 24 November 2017

Published online: 01 December 2017

\section{References}

1. Stone NN, Potters L, Davis BJ, Ciezki JP, Zelefsky MJ, Roach M, et al. Multicenter analysis of effect of high biologic effective dose on biochemical failure and survival outcomes in patients with Gleason score 7-10 prostate cancer treated with permanent prostate brachytherapy. Int J Radiat Oncol Biol Phys. 2009;73(2):341-6.

2. Taira AV, Merrick GS, Butler WM, Galbreath RW, Lief J, Adamovich E, et al. Long-term outcome for clinically localized prostate cancer treated with permanent interstitial brachytherapy. Int J Radiat Oncol Biol Phys. 2011:79(5):1336-42.

3. Nag S, Beyer D, Friedland J, Grimm P, Nath R. American Brachytherapy society (ABS) recommendations for transperineal permanent brachytherapy of prostate cancer. Int J Radiat Oncol Biol Phys. 1999;44(4):789-99.
4. Al-Qaisieh B, Carey B, Ash D, Bottomley D. The use of linked seeds eliminates lung embolization following permanent seed implantation for prostate cancer. Int J Radiat Oncol Biol Phys. 2004;59(2):397-9.

5. Fuller DB, Koziol JA, Feng AC. Prostate brachytherapy seed migration and dosimetry: analysis of stranded sources and other potential predictive factors. Brachytherapy. 2004;3(1):10-9.

6. Saibishkumar EP, Borg J, Yeung I, Cummins-Holder C, Landon A, Crook J. Sequential comparison of seed loss and prostate dosimetry of stranded seeds with loose seeds in 1251 permanent implant for low-risk prostate cancer. Int J Radiat Oncol Biol Phys. 2009;73(1):61-8.

7. Zauls AJ, Ashenafi MS, Onicescu G, Clarke HS, Marshall DT. Comparison of intraoperatively built custom linked seeds versus loose seed gun applicator technique using real-time intraoperative planning for permanent prostate brachytherapy. Int J Radiat Oncol Biol Phys. 2011;81(4):1010-6.

8. Ishiyama H, Satoh T, Kawakami S, Tsumura H, Komori S, Tabata K, et al. A prospective quasi-randomized comparison of intraoperatively built customlinked seeds versus loose seeds for prostate brachytherapy. Int J Radiat Oncol Biol Phys. 2014;90(1):134-9.

9. Stock RG, Cahlon O, Cesaretti JA, Kollmeier MA, Stone NN. Combined modality treatment in the management of high-risk prostate cancer. Int J Radiat Oncol Biol Phys. 2004:59(5):1352-9.

10. Konaka H, Egawa S, Saito S, Yorozu A, Takahashi H, Miyakoda K, et al. Trimodality therapy with 1-125 brachytherapy, external beam radiation therapy, and short- or long-term hormone therapy for high-risk localized prostate cancer (TRIP): study protocol for a phase III, multicenter, randomized, controlled trial. BMC Cancer. 2012;12:110.

11. Ohashi T, Yorozu A, Saito S, Momma T, Nishiyama T, Yamashita S, et al. Combined brachytherapy and external beam radiotherapy without adjuvant androgen deprivation therapy for high-risk prostate cancer. Radiat Oncol. 2014:9:13.

12. Saw CB, Suntharalingam N. Quantitative assessment of interstitial implants. Int J Radiat Oncol Biol Phys. 1991;20(1):135-9.

13. McLaughlin P, Narayana V, Pan C, Berri S, Troyer S, Herman J, et al. Comparison of day 0 and day 14 dosimetry for permanent prostate implants using stranded seeds. Int J Radiat Oncol Biol Phys. 2006:64(1):144-50.

14. Usmani N, Chng N, Spadinger I, Morris WJ. Lack of significant intraprostatic migration of stranded iodine-125 sources in prostate brachytherapy implants. Brachytherapy. 2011;10(4):275-85.

15. Vassiliev ON, Kudchadker RJ, Swanson DA, Bruno TL, van Vulpen M, Frank S. Displacement of periurethral stranded seeds and its dosimetric consequences in prostate brachytherapy. Brachytherapy. 2011;10(5):401-8.

16. Pinkawa M, Asadpour B, Gagel B, Piroth MD, Borchers $H$, Jakse $G$, et al. Evaluation of source displacement and dose-volume changes after permanent prostate brachytherapy with stranded seeds. Radiother Oncol. 2007:84(2):190-6

17. Chng N, Spadinger I, Rasoda R, Morris WJ, Salcudean S. Prostate brachytherapy postimplant dosimetry: seed orientation and the impact of dosimetric anisotropy in stranded implants. Med Phys. 2012:39(2):721-31.

18. Fagundes HM, Keys RJ, Wojcik MF, Radden MA, Bertelsman CG, Cavanagh WA. Transperineal TRUS-guided prostate brachytherapy using loose seeds versus RAPIDStrand: a dosimetric analysis. Brachytherapy. 2004;3(3):136-40.

19. Lin K, Lee SP, Cho JS, Reiter RE, DeMarco JJ, Solberg TD. Improvements in prostate brachytherapy dosimetry due to seed stranding. Brachytherapy. 2007:6(1):44-8.

20. Heysek RV, Gwede CK, Torres-Roca J, Cantor A, Kelley S, Saini AS, et al. A dosimetric analysis of unstranded seeds versus customized stranded seeds in transperineal interstitial permanent prostate seed brachytherapy. Brachytherapy. 2006;5(4):244-50.

21. Jarusevicius L, Inciura A, Juozaityte E, Vaiciunas K, Vaitkus A, Sniureviciute M Comparison of implant quality between loose and intra-operatively linked lodine125 seeds in prostate cancer Brachytherapy. J Radiat Res. 2012:53(3):439-46.

22. Katayama N, Takemoto M, Takamoto A, Ihara H, Katsui K, Ebara S, et al. Comparison of implant quality between intraoperatively built custom-linked seeds and loose seeds in permanent prostate brachytherapy using sector analysis. J Radiat Res. 2016:57(4):393-9.

23. Ling CC, Li WX, Anderson LL. The relative biological effectiveness of I-125 and pd-103. Int J Radiat Oncol Biol Phys. 1995:32(2):373-8.

24. Ankem MK, DeCarvalho VS, Harangozo AM, Hartanto VH, Perrotti M, Han K, et al. Implications of radioactive seed migration to the lungs after prostate brachytherapy. Urology. 2002;59(4):555-9. 
25. Eshleman JS, Davis BJ, Pisansky TM, Wilson TM, Haddock MG, King BF, et al. Radioactive seed migration to the chest after transperineal interstitial prostate brachytherapy: extraprostatic seed placement correlates with migration. Int J Radiat Oncol Biol Phys. 2004;59(2):419-25.

26. Kono Y, Kubota K, Aruga T, Ishibashi A, Morooka M, Ito K, et al. Swelling of the prostate gland by permanent Brachytherapy may affect seed migration. Jpn J Clin Oncol. 2010;40(12):1159-65.

27. Kunos CA, Resnick MI, Kinsella TJ, Ellis RJ. Migration of implanted free radioactive seeds for adenocarcinoma of the prostate using a Mick applicator. Brachytherapy. 2004;3(2):71-7.

28. Nag S, Vivekanandam S, Martinez-Monge R. Pulmonary embolization of permanently implanted radioactive palladium-103 seeds for carcinoma of the prostate. Int J Radiat Oncol Biol Phys. 1997;39(3):667-70.

29. Saibishkumar EP, Borg J, Yeung I, Cummins-Holder C, Landon A, Crook J. Sequential comparison of seed loss and prostate Dosimetry of stranded seeds with loose seeds in (125)l permanent implant for low-risk prostate cancer. Int J Radiat Oncol Biol Phys. 2009;73(1):61-8.

30. Stone NN, Stock RG. Reduction of pulmonary migration of permanent interstitial sources in patients undergoing prostate brachytherapy. Urology. 2005;66(1):119-23.

31. Sugawara A, Nakashima J, Shigematsu N, Kunieda E, Kubo A. Prediction of seed migration after transperineal interstitial prostate brachytherapy with I-125 free seeds. Brachytherapy. 2009;8(1):52-6.

32. Hirose $K$, Aoki M, Sato M, Akimoto H, Hashimoto Y, Imai A, et al. The retrospective analysis of the relationship between prescribed dose and risk factor for seed migration in iodine-125 prostate brachytherapy. Jpn J Radiol. 2016;34(11):718-23.

\section{Submit your next manuscript to BioMed Central and we will help you at every step:}

- We accept pre-submission inquiries

- Our selector tool helps you to find the most relevant journal

- We provide round the clock customer support

- Convenient online submission

- Thorough peer review

- Inclusion in PubMed and all major indexing services

- Maximum visibility for your research

Submit your manuscript at www.biomedcentral.com/submit
Biomed Central 